\title{
Kinetic Continuous Opinion Dynamics Model on Two Types of Archimedean Lattices
}

\author{
Francisco W. S. Lima* \\ Dietrich Stauffer Computational Physics Lab, Departamento de Física, Universidade Federal do Piauí, Teresina, Brazil
}

Here, the critical properties of kinetic continuous opinion dynamics model are studied on $(4,6,12)$ and $\left(4,8^{2}\right)$ Archimedean lattices. We obtain $p_{c}$ and the critical exponents from Monte Carlo simulations and finite size scaling. We found out the values of the critical points and Binder cumulant that are $p_{C}=0.086(3)$ and $O_{4}^{*}=0.59(2)$ for $(4,6,12)$; and $p_{c}=0.109(3)$ and $O_{4}^{*}=0.606(5)$ for $\left(4,8^{2}\right)$ lattices and also the exponent ratios $\beta / \nu$, $\gamma / \nu$, and $1 / v$ are, respectively: 0.23(7), 1.43(5), and 0.60(3) for $(4,6,12)$; and 0.149(4), 1.56(4), and $0.94(4)$ for $\left(4,8^{2}\right)$ lattices. Our new results disprove of the Grinstein criterion.

Keywords: Monte Carlo simulation, critical exponents, phase transition, non-equilibrium PACS numbers: 05.10.Ln, 05.70.Fh, 64.60.Fr

\section{OPEN ACCESS}

Edited by:

Antonio F. Miguel,

University of Évora, Portugal

Reviewed by:

Unjong Yu,

Gwangju Institute of Science and

Technology, South Korea

Raimundo Nogueira Costa Filho,

Federal University of Ceará, Brazil

*Correspondence:

Francisco W. S. Lima

fwslima@gmail.com

Specialty section:

This article was submitted to Interdisciplinary Physics,

a section of the journal

Frontiers in Physics

Received: 27 June 2017 Accepted: 19 September 2017 Published: 29 September 2017

Citation:

Lima FWS (2017) Kinetic Continuous

Opinion Dynamics Model on Two Types of Archimedean Lattices.

Front. Phys. 5:47.

doi: 10.3389/fphy.2017.00047

\section{INTRODUCTION}

In 1986 Galam introduced the use of local majority rules to study voting systems as bottomup democratic voting in hierarchical structures [1], see also the references [2-4]. Although sociophysics has been rejected by some physicists in the eighties [5], it is has become today an active and promising area of research for interdisciplinary physicists [6, 7].

In this same context, in 1982, de Oliveira [8] proposed a non-equilibrium model called Majority Vote Model (MVM). On two-dimensional lattices it shows critical phenomena with critical exponents $v, \beta$, and $\gamma$, as for [8-10] the equilibrium Ising model [11, 12], in agree with a hypothesis of Grinstein et al. [13].

In 2012 Biswas et al. [14] proposed a kinetic model of opinion formation. This model kinetic continuous opinion dynamics (KCOD) presents mutual interactions between the individual $i, j$ that can be both positive and negative. In this model the fraction of negative interactions is represented by a parameter $p$ in order to characterize the different types of distributions for the mutual interactions. The results of the continuous version the KCOD model, obtained through numerical simulations indicate the existence of a universal continuous phase transition at $p=p_{c}$ with exponents of mean field ( $v d=2.00(1), \beta=0.50(1)$, and $\gamma=1.00(1)$ ).

Similar to this KCOD model is the one of Deffuant et al. [15], where each person $i$ selects another person $j$ to talk to (no lattice) and both move their opinion toward that of the other person. For the Krause-Hegselmann model [16], each person averages over the opinions of the others in the population, again no lattice. While these two models use continuous opinions, those in the Sznajd model [17] usually are Ising-like $(+1$ or -1$)$ and restricted to a square lattice with four nearest neighbors. Two Sznajd people happening to agree in their opinion convince all their six neighbors of this opinion. In all these models one may start from a random distribution of opinions and then check if the opinions all converge to one consensus, two opposing camps of opinions, or a fragmentation into many opinion clusters [18-20]. 
Recently, Mukherjee and Chatterjee [21] studied the KCOD model on square and cubic lattices (2D and $3 \mathrm{D})$. Their numerical results indicate that the same critical behavior of the KCOD model and the Ising model in the corresponding dimensions.

In this work, we studied the KCOD on two Archimedean lattices-namely, $(4,6,12)$, and $\left(4,8^{2}\right)$-through extensive Monte Carlo simulations. Pictures of the $(4,6,12)$ and $\left(4,8^{2}\right) \mathrm{AL}$ are shown in Figure 1. The AL are vertex transitive graphs that can be embedded in a plane such that every face is a regular polygon. Kepler showed that there are exactly 11 such graphs. The AL are labeled according to the sizes of faces incident to a given vertex.

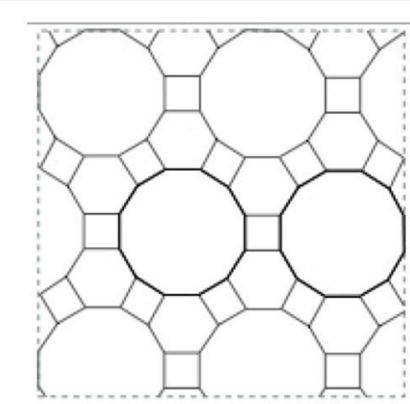

$(4,6,12)$

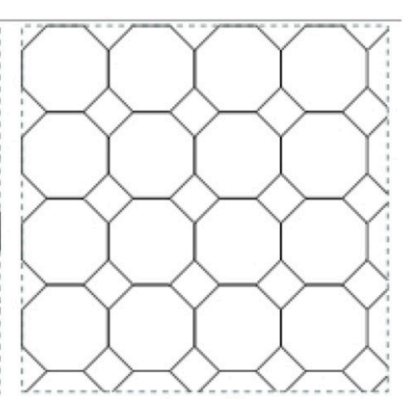

$\left(4,8^{2}\right)$
FIGURE 1 | Pictures of the $(4,6,12)$ and $\left(4,8^{2}\right) \mathrm{AL}$.
The face sizes are sorted, starting from the face for which the list is the smallest in lexicographical order. In this way, the square lattice gets the name $(4,4,4,4)$ (abbreviated to $\left(4^{4}\right)$ ), honeycomb is called $\left(6^{3}\right)$, and Kagome is $(3,6,3,6)$. Here, we also compared our results with those of the MVM made on $(3,4,6,4)$ and $\left(3^{4}, 6\right)$ AL.

One of our goals, besides finding the critical exponents of the KCOD model on $(4,6,12)$ and $\left(4,8^{2}\right) \mathrm{AL}$, is to verify the Grinstein et al. criterion [13], for non-equilibrium stochastic spin systems with up-down symmetry on $(4,6,12)$ and $\left(4,8^{2}\right)$ AL belong to the same universality class as the Ising model on $\left(4^{4}\right)$ as suggested by Grinstein et al. [13]. Here, we also compared our results with those of the MVM on $(4,6,12)$ and $\left(4,8^{2}\right)$ [22].

\section{DEFINITION AND SIMULATION}

The KCOD model [14] is defined by a set of individuals with continuous opinion variables $o_{i}(t)$, where the opinion of a person $i$ at time $t$, takes the values in the interval $[-1,+1]$, is situated on every node of the $(4,6,12)$ and $\left(4,8^{2}\right)$ AL with $N=12 L^{2}$ sites for $(4,6,12)$ and $N=4 L^{2}$ sites for $\left(4,8^{2}\right)$. In a population of $N$ individuals, opinions change out of pair-wise interactions via mutual influences/couplings $\mu_{i j}$ as:

$$
\begin{aligned}
& o_{i}(t+1)=o_{i}(t)+\mu_{i j} o_{j}(t) \\
& o_{j}(t+1)=o_{j}(t)+\mu_{i j} o_{i}(t) .
\end{aligned}
$$
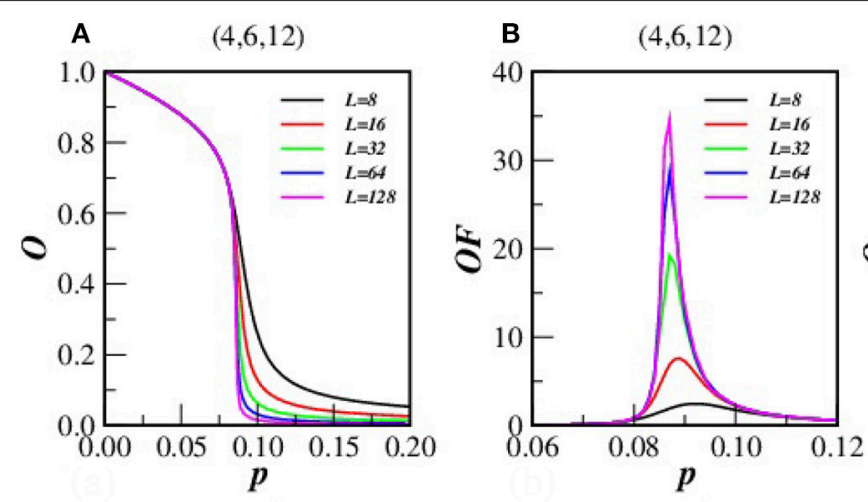

\section{C $(4,6,12)$}
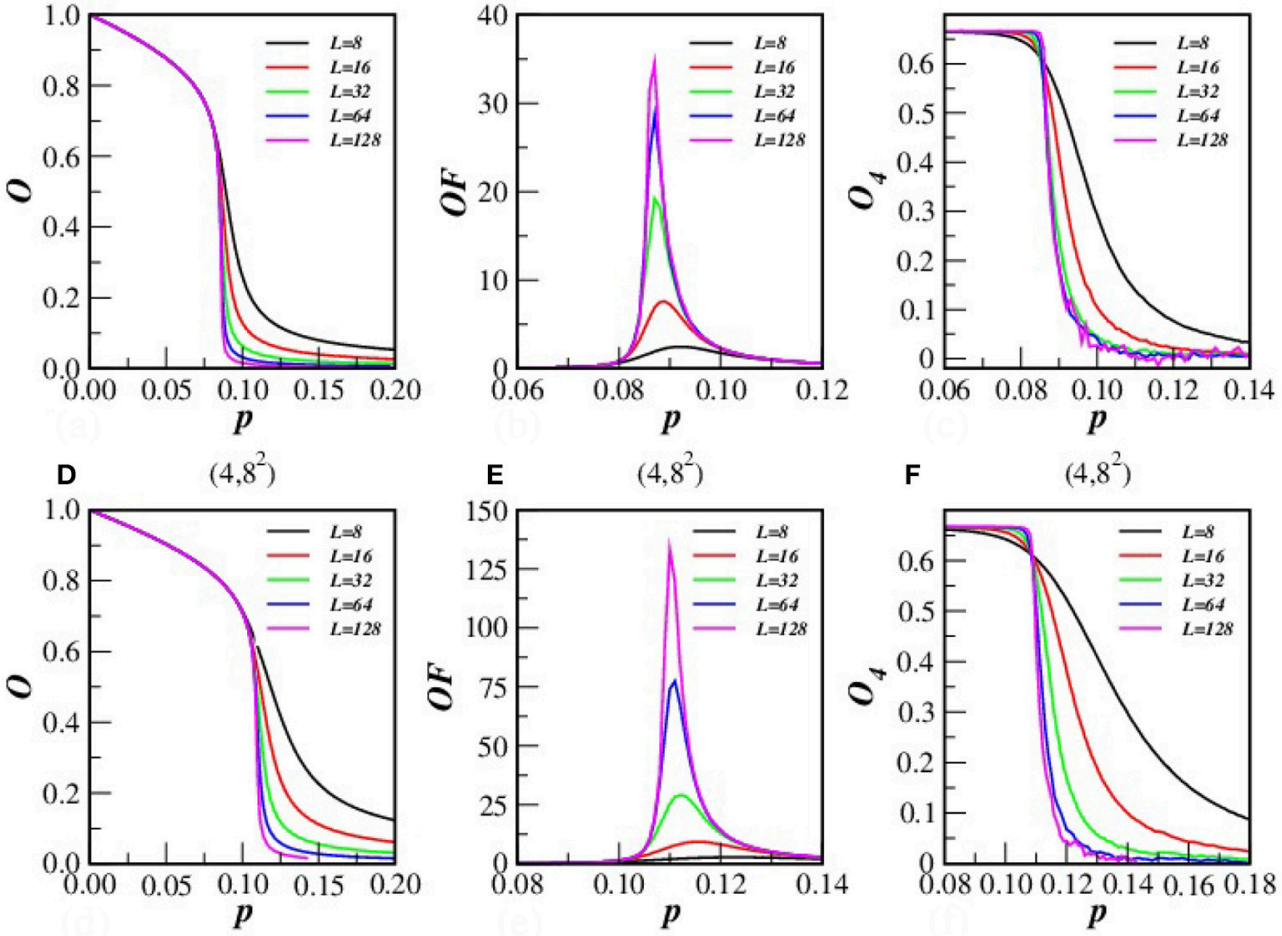

FIGURE 2 | (Color on-line) $O, O F$, and $O_{4}$ vs. $p$, for sizes $L=2^{3}, 2^{4}, 2^{5}, 2^{6}$ and $2^{7}$ and $N=12 L^{2}$ (for $4,6,12$ ) $A L$ (first line) and $N=4 L^{2}$ sites for $\left(4,8^{2}\right) A L$ (second line). 


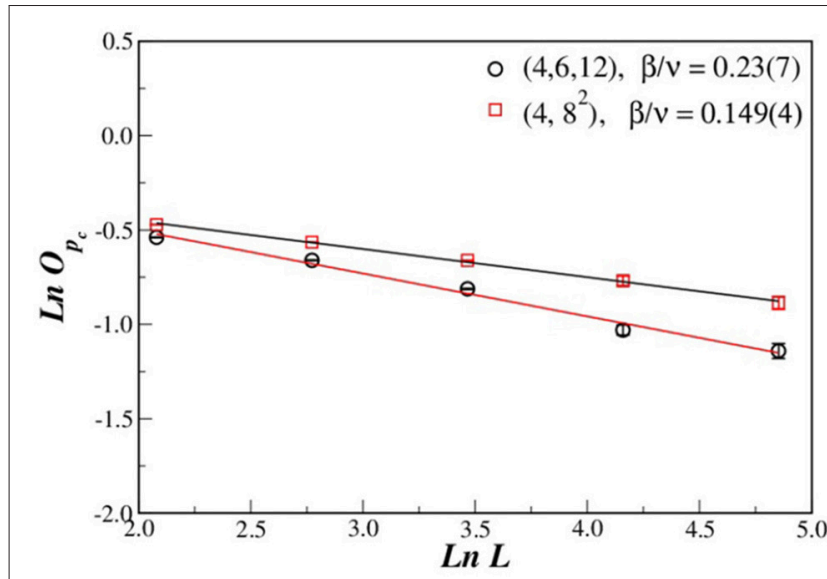

FIGURE 3 | Log-log plot of the dependence of the opinion $O^{*}=O\left(p_{C}\right)$ on the linear system size $L$. Fitting data, we obtained the estimation for the critical ratio $\beta / v$.

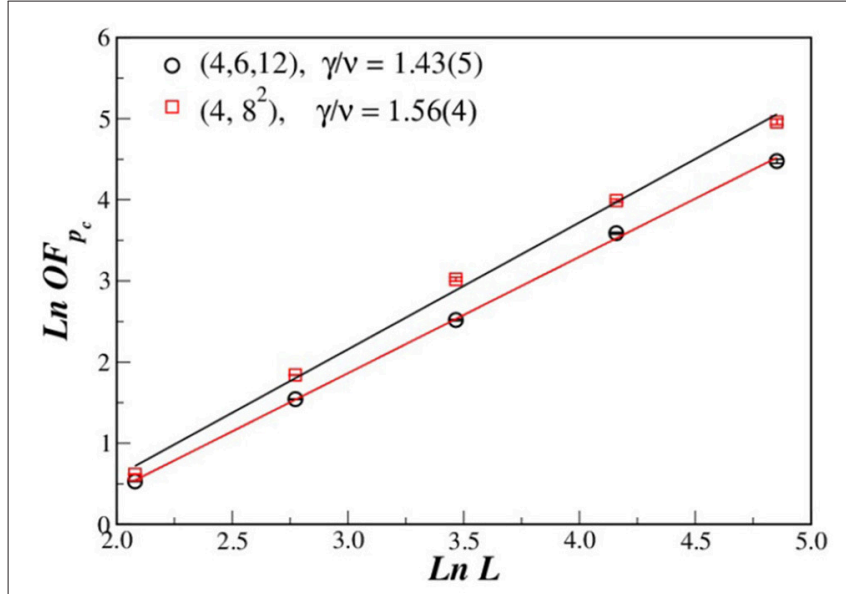

FIGURE 4 | Log-log plot of the OF at $p_{C}$ vs. $L$ for $(4,6,12)$, and $\left(4,8^{2}\right) A L$. Fitting data, we obtained the estimation for the critical ratio $\gamma / \nu$.

The pairs $i, j$ are unrestricted, meaning the original model is defined on a fully-connected graph, giving a mean-field-like limit (infinite range interactions). The pairwise interaction implies no sum over the index $j$. with real random couplings $\mu_{i j}$. Agent $i$ updates his/her opinion via Equation (1) by interacting with agent $j$ and is influenced by the coupling $\mu_{i j}$. The opinions are limited to $-1 \leq o_{i}(t) \leq 1$. If the opinion value of an individual become higher (lower) than $+1(-1)$, then it is set equal to +1 $(-1)$. This bound, along with Equation (1), defines the dynamics of the model. Here, $\mu_{i j}$ is a continuous random variable defined in the range $[-1,+1]$, i.e., it takes a random real value in the range $[-1,0]$ or $([0,1])$ with probability $p$ or $(1-p)$. In other words, the disorder parameter $p$ denotes the fraction of negative pairwise interactions. The average opinion $O=\left|\sum_{i} o_{i}\right| / N$ measures the ordering in the system. As a function of the fraction $p$ of negative interactions a symmetry breaking transition occurs between an

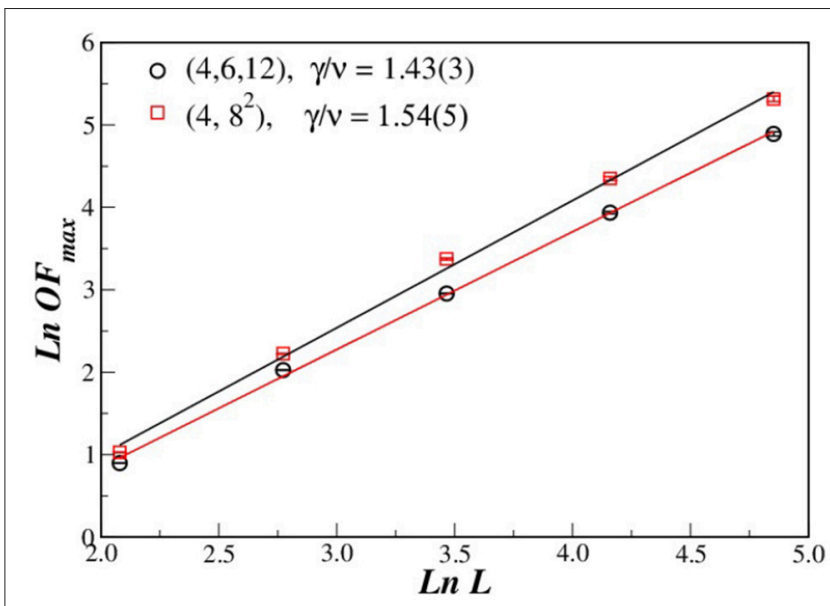

FIGURE 5 | OF at $p_{\chi \max }(N)$ vs. $L$ for $(4,6,12)$ and $\left(8^{2}\right)$, AL. Fitting data, we obtained another estimation for the critical ratio $\gamma / v$.

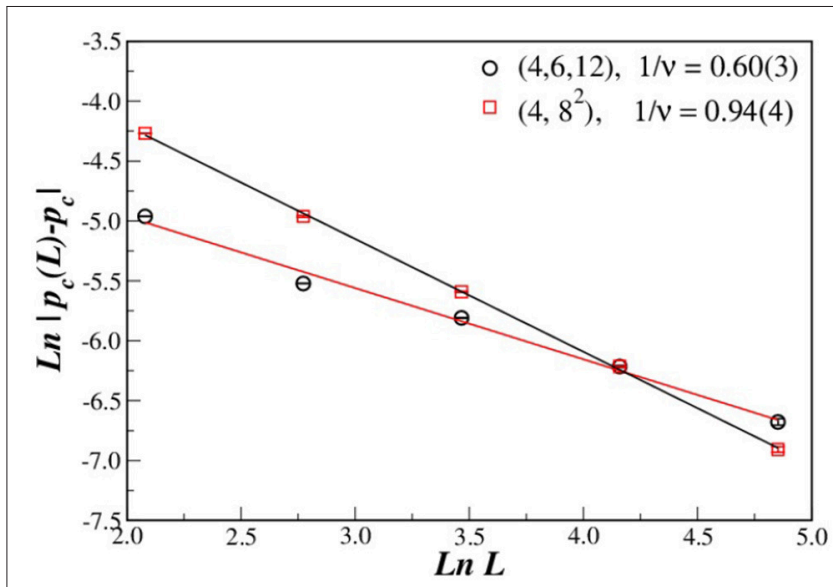

FIGURE 6 | Plot of $\ln \left|p_{C}(L)-p_{C}\right|$ vs. the linear system size $L$ for $(4,6,12)$ and $\left(4,8^{2}\right) \mathrm{AL}$. Fitting data, we obtained the estimation for the critical exponent $1 / v$.

ordered and a disordered phase: below a certain value $p_{c}$ of the parameter $p$, the system is ordered (giving a non zero value of the opinion $O$, defined in the following), while it is disordered above $p_{c}(O=0)$.

The critical properties of model we are interested in are the order parameter $O$, the order parameter fluctuations (susceptibility) OF and the reduced fourth-order cumulant of the $\mathrm{O}$, namely here by $\mathrm{O}_{4}$, defined as

$$
\begin{aligned}
O(p) & \equiv\langle O\rangle, \\
O F(p) & \equiv N\left(\left\langle O^{2}\right\rangle-\langle O\rangle^{2}\right), \\
O_{4}(p) & \equiv 1-\frac{\left\langle O^{4}\right\rangle}{3\left\langle O^{2}\right\rangle^{2}},
\end{aligned}
$$

where $\langle\cdots\rangle$ stands for time averages, computed at the steady states. $N_{\text {run }}$ independent simulations are averaged over. 

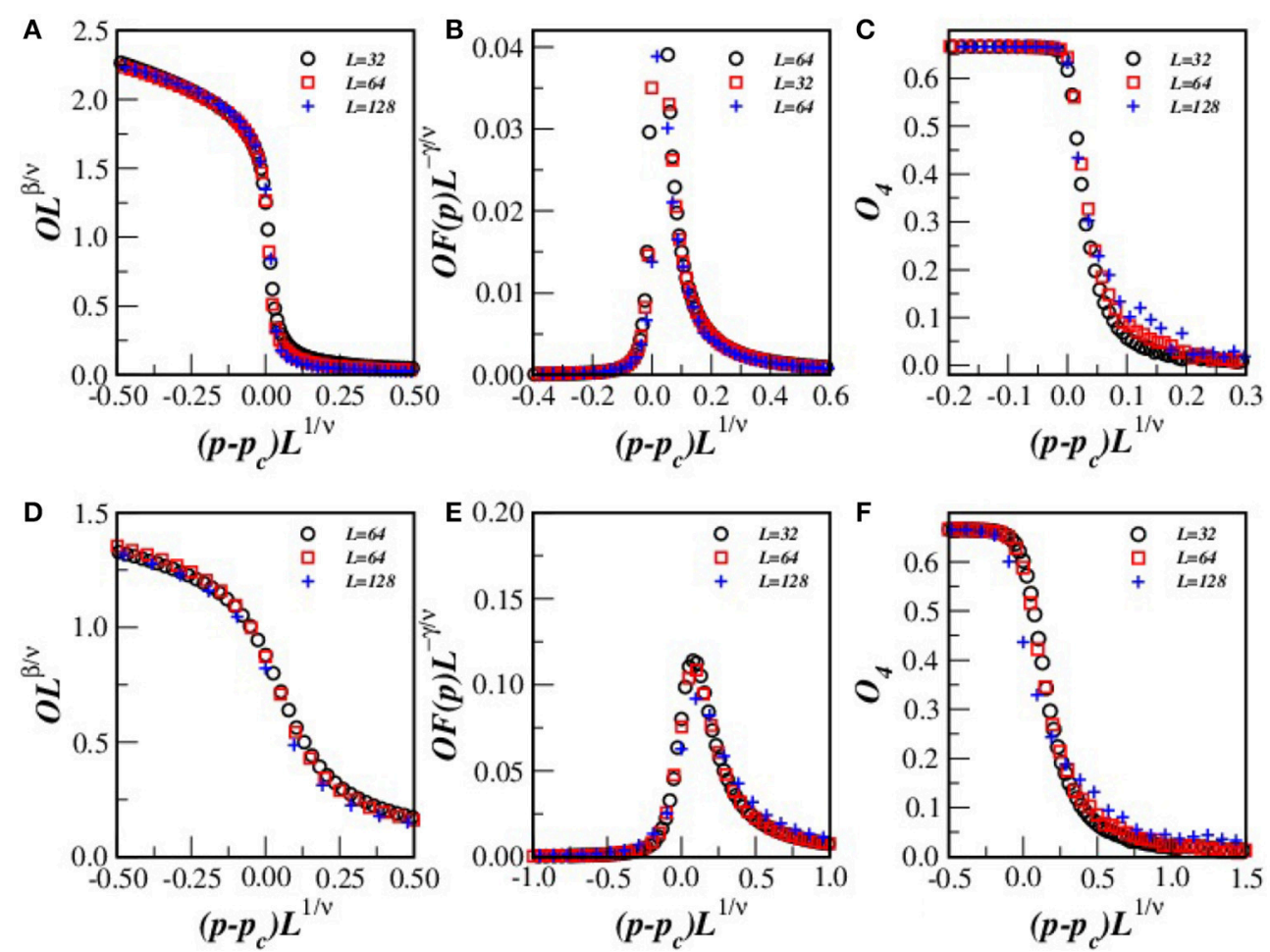

FIGURE 7 | (Color on-line) Data collapse of the opinion $O, O F$, and $O_{4}$ shown in Figure 2 for $L=32,64$, and $128(4,6,12)$ and $\left(4,8^{2}\right) \mathrm{AL}$. The exponent ratios used here were $\beta / v=0.23(7), \gamma / v=1.43(5)$, and $1 / v=0.60(3)$ for $(4,6,12)$, and $\beta / v=0.149(4), \gamma / v=1.56(4)$, and $1 / v=0.94(4)$ for $\left(4,8^{2}\right)$ AL.

TABLE 1 | Critical temperatures, exponents and effective dimensionalities for MVM on $(4,6,12)$ and $\left(4,8^{2}\right) \operatorname{AL}[22]$.

\begin{tabular}{|c|c|c|c|}
\hline MVM & $(4,6,12)$ & $\left(4,8^{2}\right)$ & $\left(4^{4}\right)$ Ising \\
\hline$T_{C}$ & 0.651 (3) & 0.667 (2) & \\
\hline$\beta / v$ & 0.105 (8) & $0.113(2)$ & 0.125 \\
\hline$\gamma / v^{T}=T_{C}$ & $1.48(11)$ & $1.60(4)$ & 1.75 \\
\hline$\gamma / v^{T=T^{*}}$ & $1.44(4)$ & $1.66(2)$ & 1.75 \\
\hline $1 / v$ & $1.16(5)$ & $0.84(6)$ & 1 \\
\hline$D_{\text {eff }}$ & $1.78(7)$ & $1.83(6)$ & 2 \\
\hline
\end{tabular}

For completeness we cite data for Ising model on $\left(4^{4}\right)$ as well [24].

The quantities $\mathrm{O}, \mathrm{OF}$, and $\mathrm{O}_{4}$ depend on the disorder parameter $p$ and obey

$$
\begin{gathered}
O=L^{-\beta / v} f_{o}(x), \\
O F=L^{\gamma / v} f_{o f}(x), \\
\frac{d O_{4}}{d p}=L^{1 / v} f_{o 4}(x),
\end{gathered}
$$

(finite-size scaling) with $\beta, \gamma$, and $v$ as the usual critical exponents, $f_{o}(x), f_{\text {of }}(x), f_{o_{4}}(x)$ as the finite-size scaling functions and

$$
x=\left(p-p_{c}\right) L^{1 / v}
$$

TABLE 2 | Critical parameter, exponents and effective dimension for KCOD model on $(4,6,12)$ and $\left(4,8^{2}\right)$.

\begin{tabular}{lccc}
\hline KCOD & $\mathbf{( 4 , 6 , 1 2 )}$ & $\mathbf{( 4 , \mathbf { 8 } ^ { 2 } )}$ & $\mathbf{( 4}^{\mathbf{4}} \mathbf{~}$ \\
\hline$p_{C}$ & $0.086(3)$ & $0.109(3)$ & $0.2266(1)$ \\
$\beta / \nu$ & $0.23(7)$ & $0.149(4)$ & $0.125(1)$ \\
$\gamma / \nu^{p=p_{C}}$ & $1.43(5)$ & $1.56(4)$ & $1.75(1)$ \\
$\gamma / \nu^{p=p^{*}}$ & $1.43(3)$ & $1.54(5)$ & \\
$1 / \nu$ & $0.60(3)$ & $0.94(4)$ & $1.01(1)$ \\
$D_{\text {eff }}$ & $1.89(6)$ & $1.76(7)$ & 2 \\
\hline
\end{tabular}

For completeness we cite data for KCOD model on (44) as well [21].

as the scaling variable. Thus, the size dependence of $O$ and $O F$ gives us the exponents $\beta / \nu$ and $\gamma / \nu$, respectively. The maximum of susceptibility also scales as $L^{\gamma / \nu}$. Moreover, the value of $p^{*}$ for which the susceptibility has a maximum scales with $L$ as

$$
p^{*}=p_{c}+b L^{-1 / v} \text { with } b \approx 1
$$

Therefore, Equations (4c) and (5) give the exponent $1 / v$. The effective dimensionality, $D_{\text {eff }}$, is given by the hyperscaling hypothesis

$$
2 \beta / v+\gamma / v=D_{\text {eff }}
$$

Monte Carlo simulations were made on $(4,6,12)$ and $\left(4,8^{2}\right)$ $\mathrm{AL}$ with various systems of size $N=768,3,072,12,288$, 
49,152, and 196,608 for $(4,6,12)$ and $N=256,1,024$, $4,096,16,384,65,536$, and for $\left(4,8^{2}\right)$ AL. We use $2 \times$ $10^{5}$ Monte Carlo steps (MCS) to make the system reach the steady state, and then the time averages are estimated over the next $3 \times 10^{5}$ MCS. One MCS is accomplished after $N$ attempts to update the opinions of agents $i$ and $j$, considering the evolution Equations (1) and (2). The results are averaged over $N_{\text {run }}\left(500 \leq N_{\text {run }} \leq 2,000\right)$ independent simulation runs for each lattice and for given set of parameters $(p, N)$.

\section{RESULTS AND DISCUSSION}

Figure 2 displays the dependence of the opinion $O, O F$, and $\mathrm{O}_{4}$ on the disorder parameter $p$, obtained from simulations on $(4,6,12)$ and $\left(4,8^{2}\right)$ AL with $N$ ranging from $N=256$ to 196,608 sites. The shape of $O(p), O F$, and $O_{4}$ curves, for a given value of $L$, indicate the occurrence of a second-order phase transition in the system. The phase transition occurs at the value of the critical disorder parameter $p_{c}$. Such critical disorder parameter $p_{c}$ is estimated as the point where the curves of the Binder cumulant $\mathrm{O}_{4}$ for different system sizes $\mathrm{N}$ intercept each other [23]. The corresponding value of $\mathrm{O}_{4}$ is represented by $O_{4}^{*}$. Then, we obtain $p_{c}=0.086(3)$ and $O_{4}^{*}=0.59(4)$; $p_{c}=0.109(3)$ and $O_{4}^{*}=0.606(5)$ for $(4,6,12)$, and $\left(4,8^{2}\right) \mathrm{AL}$, respectively.

The results obtained from Figures 3-6 and used in Figure 7 are summarized in Table 2.

The excellent curve collapses Figure 7 for distinct system sizes corroborates our estimated values for $p_{c}$ and exponent ratios $\beta / \nu$, $\gamma / \nu$ and $1 / \nu$.

The resulting critical exponents and disorder parameters are collected in Table 2. One can also see that the exponent ratios $\beta / v, \gamma / v, 1 / v$ are similar to MVM, Table $\mathbf{1}$, and are different from the Ising model contrary to the Grinstein's

\section{REFERENCES}

1. Galam S. Majority rule, hierarchical structures, and democratic totalitarianism: a statistical approach. J Math Psychol. (1986) 30:426-34; earlier Galam S, Gefen Y, Shapir Y. Sociophysics: a new approach of sociological collective behaviour. I. mean-behaviour description of a strike. J Math Sociol. (1982) 9:1-13. doi: 10.1080/0022250X.1982.99 89929

2. Galam S. Social paradoxes of majority rule voting and renormalization group. J Stat Phys. (1990) 61: 943-51. doi: 10.1007/BF01027314

3. Fortunato S, Macy M, Redner, S. Editorial. J Stat Phys. (2013) 151:1-8. doi: 10.1007/s10955-013-0703-2

4. Galam S. The drastic outcomes from voting alliances in three-party democratic voting (1990 -> 2013). J Stat Phys. (2013) 151:46-68. doi: 10.1007/s10955-012-0641-4

5. Galam S. Sociophysics: a personal testimony. Phys A (2004) 336:49-55. doi: 10.1016/j.physa.2004.01.009

6. Stauffer D. A biased review of sociophysics. J Stat Phys. (2013) 151:9-20. doi: 10.1007/s10955-012-0604-9

7. Galam S. Sociophysics: A Physicist's Modeling of Psycho-political Phenomena. New York, NY: Springer (2012). doi: 10.1007/978-1-4614-2032-3 hypothesis [13]. They are different from $\beta / \nu=0.125$ and $\gamma / \nu=1.75$ obtained for a $d=2$ lattices, but obey hyperscaling relation (within the error bars). Equation (6) yields effective dimensionality of systems $D_{\text {eff }}=1.89(6)$ for $(4,6,12)$ and $D_{\text {eff }}=1.76(7)$ for $\left(4,8^{2}\right)$. The effective dimensionalities of KCOD on our two AL are close to those for MVM on $(4,6,12) \mathrm{AL}\left(D_{\text {eff }}=1.78(7)\right)$ and on $\left(4,8^{2}\right) \mathrm{AL}$ $\left(D_{\text {eff }}=1.83(6)\right)$. The results of simulations are collected in Table 2.

\section{CONCLUSION}

We studied a non-equilibrium KCOD model through extensive Monte Carlo simulations on $(4,6,12)$ and $\left(4,8^{2}\right)$ AL. On these lattices, the KCOD model shows a second-order phase transition. Our Monte Carlo simulations suggest that the effective dimensionality $D_{\text {eff }}$ is close to two, i.e., that hyperscaling relation $2 \beta / \nu+\gamma / \nu \approx 2$ may be valid.

Finally, we remark that the critical exponents $\gamma / \nu, \beta / \nu$, and $1 / v$ for KCOD on $(4,6,12)$ and $\left(4,8^{2}\right)$ AL are similar to the MVM model on $(4,6,12)$ and $\left(4,8^{2}\right)$ AL [22], see Tables 1,2 . Therefore, this model not belong to the Ising universality class [24] and the hypothesis of Grinstein has been disproved.

\section{AUTHOR CONTRIBUTIONS}

The author confirms being the sole contributor of this work and approved it for publication.

\section{ACKNOWLEDGMENTS}

FL is grateful to Dietrich Stauffer for stimulating discussions and for critical reading of the manuscript. He also acknowledges the support the system SGI Altix 1350 the computational park CENAPAD, UNICAMP-USP, SP-BRASIL.
8. de Oliveira MJ. Isotropic majority-vote model on a square lattice. J Stat Phys. (1992) 66:273-81. doi: 10.1007/BF01060069

9. Santos MA, Teixeira S. Anisotropic voter model. J Stat Phys. (1995) 78:963-70. doi: 10.1007/BF02183696

10. Crochik L, Tomé T. Entropy production in the majority vote model. Phys Rev E (2005) 72:057103-7. doi: 10.1103/PhysRevE.72.057103

11. Hasenbusch M. Monte Carlo studies of the three-dimensional Ising model in equilibrium. Int J Mod Phys C (2001) 12:911-1009. doi: 10.1142/S0129183101002383

12. Binney JJ, Dowrick NJ, Fisher AJ, Newman, MEJ. A Theory of Critical Phenomena. An Introduction to the Renormalization Group. Oxford: Clarendon Press (1992).

13. Grinstein G, Jayaprakash C, He Y. Statistical mechanics of probabilistic cellular automata. Phys Rev Lett. (1985) 55:2527-30. doi: 10.1103/PhysRevLett.55.2527

14. Biswas S, Chatterjee A, Sen P. Disorder induced phase transition in kinetic models of opinion dynamics. Phys A (2012) 391:3257-65. doi: 10.1016/j.physa.2012.01.046

15. Deffuant G, Amblard F, Weisbuch G, Faure T. How can extremism prevail? A study based on the relative agreement interaction model. J Artif Soc Soc Simul. (2002) 5:1-26. 
16. Hegselmann R, Krause U. Opinion dynamics and bounded confidence models, analysis and simulation. J Artif Soc Soc Simul. (2002) 5:1-32.

17. Sznajd-Weron K, Sznajd J. Opinion evolution in closed community. Int J Mod Phys C (2000) 11:1157-65. doi: 10.1142/S0129183100000936

18. Costa Filho RN, Almeida MP, Andrade JS Jr, Moreira JE. Scaling behavior in a proportional voting process. Phys Rev E (1999) 60:1067-8. doi: 10.1103/PhysRevE.60.1067

19. Araripe LE, Costa Filho RN, Herrmann HJ, Andrade JS Jr. Pluraty voting: the statistical laws of democracy in Brazil. Int J Mod Phys C (2006) 17:1809-13. doi: 10.1142/S0129183106010200

20. Araripe LE, Costa Filho RN. Role of parties in the vote distribution of proportional elections. Phys A (2009) 388:4167-70. doi: 10.1016/j.physa.2009.06.023

21. Mukherjee S, Chatterjee A. Disorder induced phase transition in an opinion dynamics model: results in 2 and 3 dimensions. Phys Rev E (2016) 94:062317-22. doi: 10.1103/PhysRevE.94. 062317
22. Welington F, Lima S. Nonequilibrium model on Archimedean lattices. Cent Eur J Phys. (2014) 12:185-91. doi: 10.2478/s11534-014-0435-1

23. Binder K, Heermann DW. Monte Carlo Simulation in Statistical Physics. Berlin; Heidelberg: Springer Verlag (1988). doi: 10.1007/978-3-662-08854-8

24. Temperley HNV. Two-dimensional ising models, in: Domb C, Green MS, editors. Phase Transitions and Critical Phenomena. Vol. 1 London: Academic Press (1972).

Conflict of Interest Statement: The author declares that the research was conducted in the absence of any commercial or financial relationships that could be construed as a potential conflict of interest.

Copyright (c) 2017 Lima. This is an open-access article distributed under the terms of the Creative Commons Attribution License (CC BY). The use, distribution or reproduction in other forums is permitted, provided the original author(s) or licensor are credited and that the original publication in this journal is cited, in accordance with accepted academic practice. No use, distribution or reproduction is permitted which does not comply with these terms. 